\title{
A method to correct sampling ghosts in historic near-infrared Fourier transform spectrometer (FTS) measurements
}

\author{
S. Dohe ${ }^{1}$, V. Sherlock ${ }^{2}$, F. Hase ${ }^{1}$, M. Gisi ${ }^{1}$, J. Robinson ${ }^{2}$, E. Sepúlveda ${ }^{3,4}$, M. Schneider ${ }^{1,4}$, and T. Blumenstock ${ }^{1}$ \\ ${ }^{1}$ Karlsruhe Institute of Technology, Institute for Meteorology and Climate Research (IMK-ASF), Karlsruhe, Germany \\ ${ }^{2}$ National Institute of Water and Atmospheric Research, Lauder, New Zealand \\ ${ }^{3}$ La Laguna University, Tenerife, Spain \\ ${ }^{4}$ Izaña Atmospheric Research Center, Agencia Estatal de Meteorología (AEMET), Santa Cruz de Tenerife, Spain
}

Correspondence to: S. Dohe (susanne.dohe@kit.edu)

Received: 8 April 2013 - Published in Atmos. Meas. Tech. Discuss.: 12 April 2013

Revised: 4 July 2013 - Accepted: 9 July 2013 - Published: 13 August 2013

\begin{abstract}
The Total Carbon Column Observing Network (TCCON) has been established to provide ground-based remote sensing measurements of the column-averaged dry air mole fractions (DMF) of key greenhouse gases. To ensure network-wide consistency, biases between Fourier transform spectrometers at different sites have to be well controlled. Errors in interferogram sampling can introduce significant biases in retrievals. In this study we investigate a two-step scheme to correct these errors. In the first step the laser sampling error (LSE) is estimated by determining the sampling shift which minimises the magnitude of the signal intensity in selected, fully absorbed regions of the solar spectrum. The LSE is estimated for every day with measurements which meet certain selection criteria to derive the site-specific time series of the LSEs. In the second step, this sequence of LSEs is used to resample all the interferograms acquired at the site, and hence correct the sampling errors. Measurements acquired at the Izaña and Lauder TCCON sites are used to demonstrate the method. At both sites the sampling error histories show changes in LSE due to instrument interventions (e.g. realignment). Estimated LSEs are in good agreement with sampling errors inferred from the ratio of primary and ghost spectral signatures in optically bandpass-limited tungsten lamp spectra acquired at Lauder. The original time series of $X_{\text {air }}$ and $X_{\mathrm{CO}_{2}}\left(X_{Y}\right.$ : column-averaged DMF of the target gas $Y$ ) at both sites show discrepancies of $0.2-0.5 \%$ due to changes in the LSE associated with instrument interventions or changes in the measurement sample rate. After resampling, discrepancies are reduced to $0.1 \%$ or less at Lauder and $0.2 \%$ at Izaña. In the latter case, coincident changes in
\end{abstract}

interferometer alignment may also have contributed to the residual difference. In the future the proposed method will be used to correct historical spectra at all TCCON sites.

\section{Introduction}

Accurate prediction of future atmospheric composition and resultant radiative forcing requires a better understanding of the spatial distribution and temporal variability of the surface sources and sinks of carbon dioxide $\left(\mathrm{CO}_{2}\right)$, methane $\left(\mathrm{CH}_{4}\right)$ and other greenhouse gases (GHGs). To date, top-down trace gas surface flux inversions have been constrained by surface in situ measurements. Although the in situ measurements have high precision and absolute accuracy $(\sim 0.1 \%$, WMO, 2011), the network has inhomogeneous spatial coverage, with few or no observing stations in many regions of importance to the global carbon cycle. Furthermore, errors in modelled atmospheric transport can introduce significant errors in the surface flux estimates. Remote sensing measurements of the integrated abundance of trace gases through the atmosphere (column measurements) are less sensitive to redistribution of the trace gas due to vertical mixing. They have the potential to provide useful complementary information to constrain surface flux inversions - with quasi-global coverage in the case of satellite measurements - provided they are sufficiently precise and accurate (Miller et al., 2007; Chevallier et al., 2011; Keppel-Aleks et al., 2012).

The Total Carbon Column Observing Network (TCCON, Wunch et al., 2011, https://tccon-wiki.caltech.edu) has been 
established to provide ground-based remote sensing measurements of the column-averaged dry air mole fraction (DMF) of $\mathrm{CO}_{2}$ and other GHGs for satellite validation and carbon cycle research. It currently comprises 17 sites worldwide which routinely acquire high-resolution near-infrared (NIR) direct solar absorption spectra using Fourier transform spectrometers (FTS). Spectra are analysed using a standard algorithm to retrieve total column abundances of target GHGs and $\mathrm{O}_{2}$ and derive the GHGs column-averaged DMFs. The $\mathrm{O}_{2}$ column retrieval is used both in the derivation of the DMFs and (when combined with an accurate measurement of surface pressure) as an internal network standard (Wunch et al., 2011). Biases between FTS instruments at different sites have to be well controlled to ensure network-wide consistency of the measurements. The target inter-site comparability for TCCON using the $\mathrm{O}_{2}$ internal network standard is $0.1 \%$.

In the following we are concerned with an error source which is of special relevance for NIR FTS measurements, namely periodic errors in the $x$-axis locations where the interferogram is discretely sampled. As a result of missampling, a fraction of the input spectral signal is assigned to an incorrect frequency, generating a ghost spectrum, which can overlap with and perturb the original spectrum, leading to errors in retrieved column abundances.

Messerschmidt et al. (2010) showed that the interferogram sampling in earlier versions of the Bruker 125HR laser sampling board does not control ghost amplitudes in the NIR to the level of accuracy required for TCCON work. Typical magnitudes of the errors in $X_{\mathrm{CO}_{2}}$ and $\mathrm{O}_{2}$ column retrievals encountered in practice are $0.1-0.5 \%$, corresponding to $0.3-$ 2 ppm for $X_{\mathrm{CO}_{2}}$ (Messerschmidt et al., 2010, 2011). The FTS manufacturer Bruker Optics (Ettlingen, Germany) subsequently developed an improved laser sampling unit which offers the accuracy required by TCCON, and which has now been installed in all TCCON spectrometers. However, the post-correction of historic TCCON measurements still remains a particular problem because the sampling error varies from instrument to instrument and is also expected to change through time (e.g. on instrument intervention, change in measurement sample rate or laser power/amplitude changes) at any given site.

Messerschmidt et al. (2010) proposed an empirical ghost correction scheme which is based on (1) the determination of the ghost-to-parent intensity ratio (GPR) using narrowband lamp spectra and (2) an empirical determination of the variation of the column-averaged DMF $X_{Y}$ of the target gas $Y$ as a function of the GPR. However, there are several reasons the scheme described by Messerschmidt et al. (2010) cannot be applied satisfactorily in practice to historical TCCON time series. Firstly, the retrieval bias depends on the sign and magnitude of the sampling error, but only the latter can be determined from the lamp GPR measurement. Secondly, a site-independent relationship between $X_{Y}$ and GPR cannot generally be assumed, due to differences in the instrument spectral response and/or differences in the absorber line-ofsight abundances (which impact both the magnitude of the aliased ghost signatures and the retrieval sensitivity to these artifacts). Thirdly, no TCCON site has a set of narrowband filter lamp spectra acquired through time which would enable changes in the sampling error on instrument intervention to be adequately characterised (even if the sign of the sampling error and the retrieval sensitivity could be adequately determined/modelled).

In this work we introduce a fundamental ghost correction scheme for historic measurements. The method consists of two steps: in the first step the degree of missampling is determined from a subset of suitable interferograms. This assumes the kind of missampling under consideration here is a slowly varying function of instrumental status (unless an intervention on the spectrometer is performed), so a subset of interferograms can be used to reconstruct the full history of laser sampling errors (LSE). In the second step, all interferograms of the time series are resampled and retransformed, and the corrected spectra are reanalysed. We demonstrate the proposed method using time series of measurements acquired at the TCCON sites in Izaña and Lauder. The following section describes the instrumentation at both sites and the data analysis within TCCON. Section 3 deals with the theory of ghosts and explains the strategy of ghost correction of historic measurements in detail. The results are presented in Sect. 4, and Sect. 5 summarises and concludes the study.

\section{Instrumentation and data analysis}

\subsection{TCCON data acquisition}

NIR measurements at both case study sites are undertaken in accordance with the TCCON data protocol (https:// tccon-wiki.caltech.edu/Network_Policy/Data_Protocol). NIR solar absorption spectra are measured with a Bruker 125HR FTS using a DC-enabled room temperature InGaAs detector with a resolution of $0.02 \mathrm{~cm}^{-1}$ (maximum optical path difference $45 \mathrm{~cm}$ ) and high folding limit of $15798 \mathrm{~cm}^{-1}$.

Since the work of Messerschmidt et al. (2010) there have been several versions of the Bruker 125HR laser sampling unit. Their laser sampling error characteristics are summarised here briefly for reference. Prior to board version ECL03 the LSE could not be minimised and was a function of sample rate (scanner velocity). At version ECL03 a potentiometer was introduced enabling the LSE to be minimised for a given sample rate (sampling errors at other sample rates remained significant). Version ECL04 of the laser board and improved linearity of the v02 laser diodes have reduced the magnitude of the LSE. The LSE is still manually minimised via a potentiometer adjustment, but once minimised, the residual sample rate dependence is generally negligible for practical purposes. The ECL05 board has a modification to further minimise any temperature dependence of 
the LSE. The ECL04/05 boards and v02 laser diodes were distributed by Bruker to TCCON partners in 2010 and installed over the course of the following year.

\subsection{Case study sites}

\subsubsection{Izaña}

The Izaña Atmospheric Research Center is a subtropical high-mountain observatory on Tenerife $\left(28.3^{\circ} \mathrm{N}, 16.5^{\circ} \mathrm{W}\right.$, Canary Islands, Spain) located at $2370 \mathrm{~m}$ altitude over a temperature inversion layer, acting as a natural barrier for local pollution. The site provides an extensive measurement programme, which includes both in situ and ground-based FTS measurements. The latter were started in the late 1990s in collaboration between the Meteorological State Agency of Spain (AEMET) and the Karlsruhe Institute of Technology (KIT). The FTS programme is involved in two global networks: for measurements in the mid-infrared (MIR) region it has contributed to the Network for the Detection of Atmospheric Composition Change (NDACC, http://www. ndacc.org) since 1999 and from 2007 also to TCCON (http: //www.tccon.caltech.edu) for NIR measurements.

The Izaña TCCON measurements are acquired with the Bruker 125HR spectrometer, which had the original ECL02 laser board at installation (January 2005). In June 2008 the instrument was realigned and the interferogram recording mode was changed from AC to DC. The electrical lowpass filter was changed in March 2009. In November 2009 the laser board was replaced by an ECL03 board (LSE was minimised at $40 \mathrm{kHz}$ ) and an optical long-pass filter was installed. After some problems due to loss of power and very cold temperatures inside the container (JanuaryMarch 2010) and the change of the electrical low-pass filter (May 2010), the new ECL04 laser board and v02 diodes were installed in November 2010 and the LSE was eliminated for all practical purposes in routine TCCON measurements. The interferometer was also realigned at this time. All Izaña measurements are acquired with a $40 \mathrm{kHz}$ sample rate.

Izaña NIR interferograms are recorded using the Optics User Software (OPUS) version 6.5 provided by Bruker. Izaña datasets presented in this paper have been analysed in a selfconsistent manner using software developed at Karlsruhe Insitute of Technology by F. Hase, M. Gisi and M. Schneider to determine the sampling error (ghost4.exe) and resample and transform interferograms to spectra. Retrievals were performed with the PROFFITT software (Hase et al., 2004).

\subsubsection{Lauder}

Lauder is located in a sparsely populated rural environment on the South Island of New Zealand $\left(45.0^{\circ} \mathrm{S}, 169.7^{\circ} \mathrm{E}\right)$ $370 \mathrm{~m}$ a.s.l. (above sea level). It is a long-established atmospheric monitoring station with an MIR FTS measurement programme which has contributed to the NDACC since 1990
(Griffith et al., 2003) and NIR FTS measurements within the TCCON since 2004.

The Lauder TCCON measurements from 2004 to 2010 were acquired with an older $120 \mathrm{HR}$ spectrometer. In August 2009 a 125HR spectrometer was installed at the site and commissioned over the following six months. The LSE characterisation in this paper is restricted to the $125 \mathrm{HR}$ time series.

At installation the Lauder 125HR spectrometer had an ECL03 laser board and the LSE was minimised at $20 \mathrm{kHz}$. At the time, the sample rate dependence of the LSE minimisation for the ECL03 boards was not fully appreciated. The early operational data (November 2009-January 2010) were acquired with a $10 \mathrm{kHz}$ sample rate and require ghost correction. From February 2010 to January 2011 routine measurements were acquired with a $20 \mathrm{kHz}$ sample rate and LSEs were small. In January 2011 the replacement laser board (ECL05) and diodes provided by Bruker were installed and the LSE reminimised for the $20 \mathrm{kHz}$ sample rate used in routine measurements.

In addition to routine solar measurements, a number of experimental datasets were acquired on the Lauder 125HR, which can be used to validate the resampling algorithm. These experiments are (1) solar measurements cycling through the 10, 20 and $40 \mathrm{kHz}$ sample rates, typically undertaken around local noon, and (2) tungsten lamp spectra acquired with an optical filter used to characterise the LSE at the three sample rates following the procedure proposed by Messerschmidt et al. (2010), and described in more detail in Sect. 3.4 (Lamp LSE estimates).

Lauder NIR measurements are acquired using OPUS version 6.5. Lauder data presented in this paper are analysed in a self-consistent manner using the standard TCCON data-processing software: IPP to transform interferograms to spectra, and GGG for TCCON retrievals. Code to determine the LSE (resample-opus) has been written based on IPP, and results are in excellent agreement with ghost4.exe. The resampling algorithm has been implemented as an additional subroutine call in the IPP software. These software developments will be adopted in standard TCCON processing in the future.

\subsection{Trace gas retrievals}

The column-averaged dry air mole fraction (DMF) of the target trace gas $Y$ is derived from the retrieved total columns $C$ of $Y$ and $\mathrm{O}_{2}$ :

$X_{Y}=0.2095 \frac{C_{Y}}{C_{\mathrm{O}_{2}}}$,

assuming a constant dry air mole fraction of 0.2095 for $\mathrm{O}_{2}$. In this paper we characterise the error in the primary TCCON parameter, $X_{\mathrm{CO}_{2}}$. Following standard TCCON procedure, the $\mathrm{CO}_{2}$ column is retrieved separately for the two bands centred at 6228 and $6348 \mathrm{~cm}^{-1}$, and averaged to calculate the $\mathrm{CO}_{2}$ 
column. The $\mathrm{O}_{2}$ column is analysed from the band centred at $7882 \mathrm{~cm}^{-1}\left(a^{1} \Delta_{g}-X^{3} \sum_{g}^{-}(0,0)\right)$.

The accuracy of $X_{Y}$ is improved by taking the ratio with the retrieved $\mathrm{O}_{2}$ column because systematic errors that are common to target and $\mathrm{O}_{2}$ column retrievals (e.g. tracker pointing errors, instrument line shape errors, zero level offsets) compensate in the ratio. The LSE gives rise to systematic errors which are site and time dependent. The errors are more important for the $\mathrm{O}_{2}$ retrieval (Messerschmidt et al., 2010) and do not cancel in the $X_{Y}$ ratio.

We will use the variable $X_{\text {air }}$ to characterise the contribution of $\mathrm{O}_{2}$ retrieval errors in a way that is readily understood. $X_{\text {air }}$ is the column average dry air mole fraction of dry air,

$X_{\text {air }}=0.2095 \frac{C_{\text {air }}\left(C_{\mathrm{H}_{2} \mathrm{O}}, P_{\mathrm{s}}\right)}{C_{\mathrm{O}_{2}}}$,

where the dry air column abundance in the numerator is derived from an independent co-located measurement of surface pressure $\left(P_{\mathrm{S}}\right)$, corrected for the $\mathrm{H}_{2} \mathrm{O}$ column overburden. In the absence of any systematic errors, the expected value of $X_{\text {air }}$ is 1 , and the relative error in $X_{Y}$ due to $\mathrm{O}_{2}$ retrieval errors is calculated/given by $X_{\text {air }}-1$.

In the presence of LSE, the records of retrieved $\mathrm{O}_{2}$ columns are biased, whereas the independent measurement of $P_{\mathrm{S}}$ remains unaffected. Stepwise changes in the LSE on instrument intervention introduce discontinuities in the $X_{\text {air }}$ time series. Other measurement and retrieval error sources (timing and pointing errors, instrument line shape (ILS) and spectroscopic errors) can introduce changes in $X_{\text {air }}$, so $X_{\text {air }}$ can only be exploited to characterise the LSE history if these other factors are controlled well enough. Significant effort has been invested at both sites to ensure this is the case, including the development of the camera-controlled highprecision solar tracker system CamTracker (Gisi et al., 2011) used at Izaña and regular monitoring of the ILS by LINEFIT analysis of low-pressure $\mathrm{HCl}, \mathrm{HBr}$ and $\mathrm{N}_{2} \mathrm{O}$ cell spectra (Hase et al., 1999). Solar zenith angle ranges are restricted in some analyses to prevent changes in $X_{\text {air }}$ of instrumental origin from being masked by air-mass-dependent retrieval biases due to spectroscopic errors.

\section{Periodic sampling errors and sampling ghosts}

\subsection{Theory of ghost formation}

Interferograms recorded with FTS need to be sampled very accurately as a function of optical path difference (OPD) to avoid artificial spectral lines (ghosts) after Fourier transformation (FT). To ensure this precise sampling, a frequencystabilised, single-mode HeNe reference laser is fed into the spectrometer. The zero crossings of the laser interferogram serve as reference for the sampling of the infrared signal. The original measurement signal of the reference laser is actually a cosine oscillation. If the construction of the mean intensity level is erroneous, a sampling error pattern of alternating narrow and wide distances will result (see Fig. 1).

Following Guelachvili (1981), the spectrum of monochromatic light derived from an interferogram with periodic sampling error $\epsilon$ of frequency $\beta$ and amplitude $\epsilon_{0}$,

$\epsilon=\epsilon_{0} \sin (2 \pi \beta x)$

is given by

$$
\begin{aligned}
B(\sigma) & =J_{0}\left(2 \pi \sigma_{0} \epsilon_{0}\right) \int_{0}^{x_{m}} \sin \left(2 \pi \sigma_{0} x\right) \sin (2 \pi \sigma x) \mathrm{d} x \\
& +\sum_{k=1}^{\infty} J_{k}\left(2 \pi \sigma_{0} \epsilon_{0}\right) \int_{0}^{x_{m}}\left[\sin \left(2 \pi\left(\sigma_{0}+k \beta\right) x\right)\right. \\
& \left.+(-1)^{k} \sin \left(2 \pi\left(\sigma_{0}-k \beta\right) x\right)\right] \sin (2 \pi \sigma x) \mathrm{d} x,
\end{aligned}
$$

where $x$ is the OPD, $\sigma_{0}$ is the frequency of the incident light and $J_{k}$ are Bessel's functions for integers $k=1,2, \ldots, n$. The $k=0$ term describes the incident radiation modified by the instrument response function for finite maximum optical path difference $x_{\mathrm{m}}$. If $\epsilon_{0} \neq 0$ the intensity of the parent line is scaled by the factor $J_{0}\left(2 \pi \sigma_{0} \epsilon_{0}\right)$ which is less than one. Similar features with intensities proportional to $J_{1}\left(2 \pi \sigma_{0} \epsilon_{0}\right), J_{2}\left(2 \pi \sigma_{0} \epsilon_{0}\right), \ldots J_{n}\left(2 \pi \sigma_{0} \epsilon_{0}\right)$ appear at $\sigma_{0} \pm \beta, \pm 2 \beta, \ldots, \pm n \beta$.

$\beta$ depends on the sampling rate per laser wavelength, which is determined by the high folding limit (HFL) for the measurements. In the case of TCCON measurements, the HFL is set to $15798 \mathrm{~cm}^{-1}$ and the laser interferogram is sampled on every zero crossing. Thus $\beta=15798 \mathrm{~cm}^{-1}$ and ghosts occurring at $\sigma_{0} \pm 15798 \mathrm{~cm}^{-1}$ are both aliased to $15798-\sigma_{0} \mathrm{~cm}^{-1}$. Note FTS measurements in the MIR as acquired by the NDACC are not affected by this problem. In this spectral region a high folding limit of $7899 \mathrm{~cm}^{-1}$ or less is applied. The sampling is performed only at each rising zero crossing of the laser interferogram, and an error in the evaluation of the mean laser intensity level does not generate an alternating sampling pattern.

In summary, a periodic sampling error leads to distortions of the true spectrum. Line strengths are modified and the spectrum zero levels become spuriously nonzero. These distortions can and do lead to significant retrieval biases in the case of TCCON measurements. They must be corrected at the source by resampling the interferograms.

\subsection{Quantification of the sampling error}

\subsubsection{Interferogram resampling}

In the interferogram resampling method we seek to determine the sampling shift which minimises the magnitude of the signal intensity in selected, fully absorbed regions of the solar spectrum. In this study we use the $7290-7360 \mathrm{~cm}^{-1}$ interval shown in Fig. 2. It is essentially opaque for 


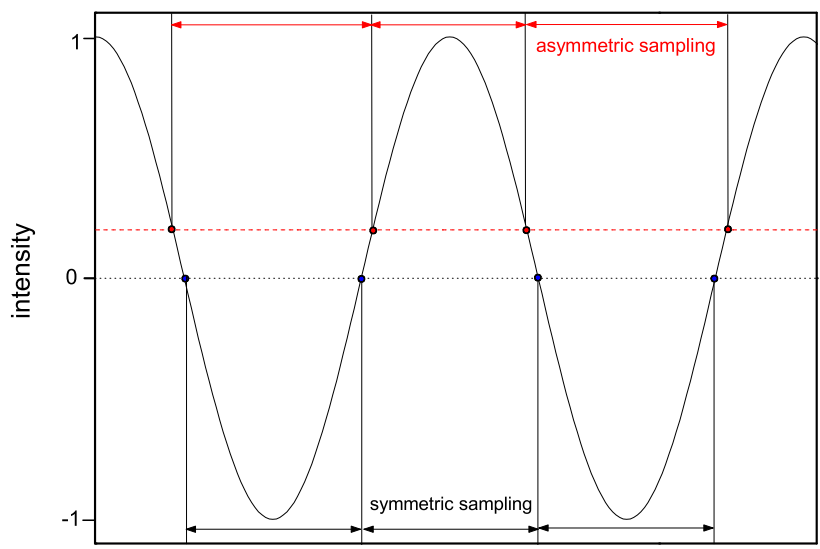

Fig. 1. An interferogram of the reference laser. The zero crossings are taken as reference for the measurement sampling which is ideally symmetric (black arrows). Due to an erroneous determination of the mean intensity level, a zero offset occurs, which gives rise to an asymmetric sampling (red arrows). As a consequence phase ghosts arise, which are added to the spectrum.

slant $\mathrm{H}_{2} \mathrm{O}$ column abundance of $>5 \times 10^{22}$ molecules $\mathrm{cm}^{-2}$ (generally satisfied at large solar zenith angles), and the associated aliased ghost interval at $8440-8510 \mathrm{~cm}^{-1}$ $\left(\mathrm{HFL}=15798 \mathrm{~cm}^{-1}\right)$ has appreciable signal levels. Izaña is unique among the TCCON sites due to its high altitude, and often the $7290-7360 \mathrm{~cm}^{-1}$ microwindow is not saturated. Therefore a second microwindow $\left(10900-11300 \mathrm{~cm}^{-1}\right)$ was used in addition to the $7290-7360 \mathrm{~cm}^{-1}$ microwindow to determine the LSE time series at Izaña (the results for these two microwindows are shown with different symbols in Fig. 6).

The sampling error is determined using the low-resolution double-sided interferogram portion of the high-resolution interferogram. A set of probable sampling errors $\Delta x_{i}$ is defined. For each $\Delta x_{i}$ the odd sample points of the interferogram are shifted and the interferogram $(I(x))$ is interpolated to the shifted sample locations assuming

$I(x+\Delta x) \simeq I(x)+\frac{\partial I}{\partial x} \Delta x$.

The derivative of the interferogram with respect to the sampling position $x$ is estimated by determining the local slope of the interferogram at each odd sample location using a sinc interpolation. We then take the FT of the resampled interferogram and evaluate the mean absolute signal intensity $\left(\sum|B(\sigma)|\right)$ in the selected spectral window (7290$7360 \mathrm{~cm}^{-1}$ ). The signal intensity is tabulated as a function of $\Delta x$, and the shift which minimises the mean absolute signal intensity is the LSE used subsequently to correct the full interferogram. The procedure is performed separately for the forward and reverse scans to allow for differences in magnitude as well as in sign between LSE of the two scan directions. Example output from a resampling test for the (7290$7360 \mathrm{~cm}^{-1}$ ) is shown in Fig. 3. Here and throughout the

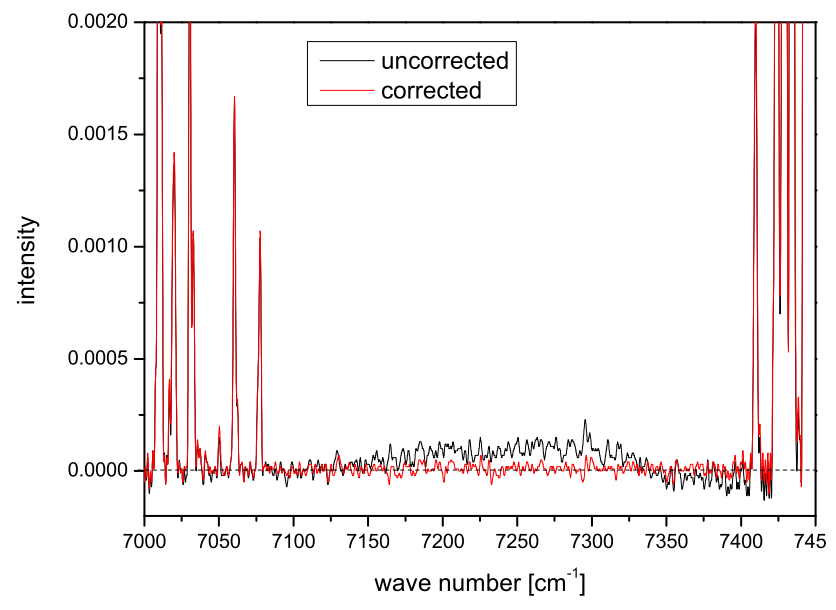

Fig. 2. The opaque region of the solar spectrum between 7290 and $7360 \mathrm{~cm}^{-1}$ used in the resampling analysis. The mean intensity in the modulus spectrum is reduced when the ghost correction is applied (black: uncorrected; red: corrected).

paper the sampling error is expressed as a fraction of the sampling interval.

As noted above, the interferogram sampling points are classified into even and odd points. The phase of the laser interferogram changes by $\pi$ with a one-point indexing shift, and the inferred LSE is of opposite sign. The classification or indexing used to derive the LSE must therefore be consistent with the indexing used when applying the LSE to correct interferograms. Although the laser fringe counting of the Bruker spectrometer is performed in quadrature, and therefore assures that the sampling number of a physical point is conserved, this assignment is lost during re-initialisation of the scanner or if the spectrometer is switched off. On the other hand, the true physical location of the infrared beam zero path difference (ZPD) constructive interference maxima (centreburst peak) and the phase of the laser interferogram are expected to remain constant through scanner reinitialisation etc. For this reason, it is preferable for our purpose to reference the sample indexing to the centreburst peak position. The centreburst of a real interferogram is not perfectly symmetric. The shapes of forward and backward centrebursts differ due to small time delays between IR and laser signal-processing chains which depend on scanner speed, optical bandpass and electrical filter settings. So it can happen that the even/odd referencing differs for forward and reverse scans and may switch upon instrumental intervention.

In its current implementation the resampling LSE estimate is based on the minimisation of the mean absolute signal intensity in an opaque section of the atmospheric spectrum. This assumes that detector nonlinearity is negligible or has been corrected prior to the LSE estimation procedure.

The current implementation also assumes that the LSE determined at the position around the interferogram centrebursts are representative for the whole high-resolution 


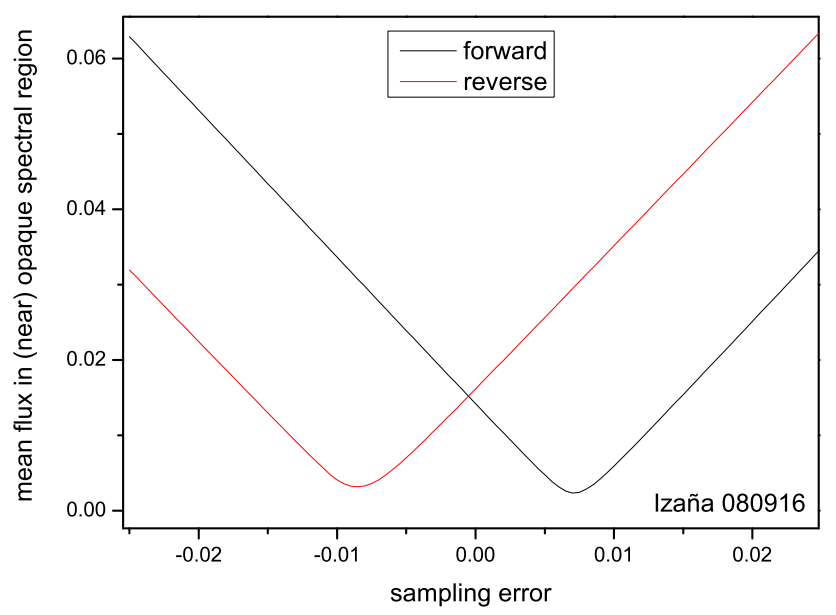

Fig. 3. The mean flux in the (near) opaque spectral region for the spectral window $7290-7360 \mathrm{~cm}^{-1}$ as a function of LSE. The scanner velocity of $v=40 \mathrm{kHz}$ introduces a significant missampling for forward and reverse scans.

interferogram. This is probably not fully verified, so some high-resolution artifacts of the original ghosts might survive. However, it would be difficult to characterise these tiny artifacts and to use them for an improved correction scheme (in which the LSE would become a function of optical path difference), because the offset introduced by the ghosts is significantly lower than the noise level of the high-resolution spectrum.

One known source of short-term variability in the LSE is not addressed by the current implementation of the resampling methodology. The LSE can vary significantly at very low pressure $(<0.5 \mathrm{hPa})$ due to heating of the electronic components of the laser board (Ralf Sussmann, Markus Rettinger, Axel Keens, personal communication, 2011). In this case, the LSE must be determined at higher temporal resolution if possible. Where this is not possible, affected historical data should not be retained for TCCON analysis, or should be flagged as ghost affected. The temperature dependence of the LSE in the ECL04 board is negligible because the LSE itself is acceptably small. DC power dissipation has been minimised in the ECL05 board, thus minimising the temperature dependence of the residual LSE.

\subsubsection{Derivation of the LSE time series}

In order to establish a time series of LSE estimates for a given site, we assume the LSE is stable or slowly varying on timescales of days to weeks, but allow for possible, discontinuous changes in the LSE during instrument intervention (depending on the nature of the intervention).

Interferograms are selected which were acquired in cloudfree conditions at large solar zenith angles to ensure the optical opacity of the $7290-7360 \mathrm{~cm}^{-1}$ microwindow. All selected interferograms acquired on the same day are coadded, and the LSE is determined using the resampling algorithm on the coadded interferogram.

Selection criteria for the Lauder time series analysis are solar zenith angles in the range $60-75^{\circ}$, line-of-site (LOS) $\mathrm{H}_{2} \mathrm{O}$ column abundance of $>5 \times 10^{22}$ molecules $\mathrm{cm}^{-2}$ and coadd sample size $\geq 10$. The resulting time series comprises 240 days over two years.

In the case of Izaña 28 days with clear-sky conditions were selected. For every day, measurements with large SZA were coadded (coadd sample size $\geq 4$ interferograms). In the case of only few measurements with large SZA on a single day, measurements of several days were coadded and the same LSE value is reported for each of these days.

\subsection{Correction of the sampling error}

The LSE time series is evaluated to define the most suitable temporal description of the LSE to apply to all measurements acquired at the given site. Typically this will be a piecewise linear function with a constant LSE for stable periods between instrument interventions. Each full-resolution interferogram is corrected based on the LSE estimate for the given day. The odd sample points of the full-resolution interferogram are shifted and the interferogram $(I(x))$ is interpolated to the shifted sample locations as previously (Eq. 5). The resampled interferogram is transformed (FT) and retrievals are performed for the resultant, ghost-corrected spectrum.

\subsection{Lamp LSE estimates}

Bandpass-limited lamp spectra were acquired at Lauder using two optical filters, referred to hereafter as the NDACC and TCCON filters, to determine the GPR following Messerschmidt et al. (2010). The NDACC filter spans a $100 \mathrm{~cm}^{-1}$ interval centred on $4150 \mathrm{~cm}^{-1}$, with ghost alias at 11598.0 $11698.0 \mathrm{~cm}^{-1}$. The TCCON filter spans a $200 \mathrm{~cm}^{-1}$ interval centred on $5790 \mathrm{~cm}^{-1}$ with ghost alias at 9908.0 $10108.0 \mathrm{~cm}^{-1}$. The GPR is calculated by the ratio of the signal intensities at the parent and ghost frequencies integrated across the filter bandpass. The GPR is wavenumber dependent and is related to the LSE by a factor $\gamma=(\pi \sigma \Delta)^{-1}$, where $\sigma$ is the optical filter bandpass centre frequency, and $\Delta$ is the sampling interval (Learner et al., 1996).

The resampling algorithm was also applied to the lamp spectra. In this case, the magnitude of the signal intensity is minimised for the ghost alias wavenumber interval of the respective filter. 


\section{Results}

\subsection{LSE time series}

\subsubsection{Lauder 125HR time series}

The LSE time series derived from solar interferograms for the Lauder 125HR is illustrated in Fig. 4. Corresponding LSE estimates determined from analysis of tungsten lamp spectra acquired with narrow bandpass optical filters are illustrated in Fig. 5. A comparison of lamp and solar LSE and GPR estimates is given in Table 1 .

Consider first the resampling results derived from solar data acquired with the original ECL03 laser board and v01 diodes (dates prior to January 2011). The LSE estimates for forward and reverse scans are comparable in magnitude but opposite in sign. The corresponding retrieval biases (Sect. 4.2) are independent of scan direction, indicating this is a result of the sample point indexing definition (specifically, forward and reverse scan peak positions differ by one point).

The LSE for the ECL03 board are sample rate dependent, as expected. The LSE is minimum for the $20 \mathrm{kHz}$ data because the sampling error was minimised for this sample rate. Stepwise changes in the $20 \mathrm{kHz}$ LSE are evident for the early instrument interventions, and a drift (or possibly a further stepwise change) is apparent during the period July 2010January 2011. The magnitude of the $10 \mathrm{kHz}$ LSE derived from solar measurements appears fairly stable through time. There is a slight increase in the LSE following the instrument intervention in December 2009. The magnitude of LSE is greatest at $40 \mathrm{kHz}$, and the sign of the LSE for a given scan direction has opposite sign to that at 10 and $20 \mathrm{kHz}$. This sign change is not an indexing artifact and is discussed further in Sect. 4.2.1.

Lamp LSE estimates derived with NDACC and TCCON filters are in excellent agreement with one another and with the sampling error inferred from the GPR estimate proposed by Messerschmidt et al. (2010). The scan direction and sample rate dependence of the signs of the LSE determined from lamp measurements with the NDACC optical filter are consistent with those determined from the solar measurements up to a global sign change of -1 (which is attributed to differences in determining the ZPD index for the wider lamp interferogram centreburst). The sign of the LSE determined using the TCCON filter is more difficult to interpret, but once differences in ZPD indexing for different scan directions and sample rates are taken into account, the sample rate dependence of the sign of the LSE is consistent with that inferred from solar data and lamp measurements with the NDACC filter.

There is good qualitative agreement between the magnitude of ECL03 lamp and solar LSE estimates at the three sample rates. Solar and lamp estimates at $10 \mathrm{kHz}$ are in excellent agreement; however, there are differences of up

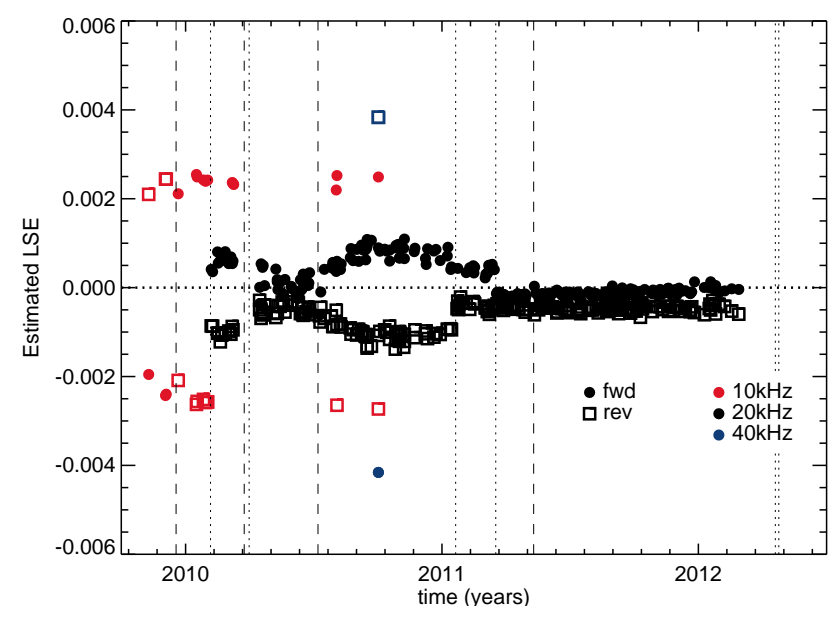

Fig. 4. Lauder 125HR LSE time series determined from resampling analysis of solar interferograms using the $7290-7360 \mathrm{~cm}^{-1}$ interval. Instrument changes relevant to the LSE and/or its determination are shown with dashed lines: change from 10 to $20 \mathrm{kHz}$ sample rate for routine acquisition (February 2010), increased phase resolution (April 2010), laser board and diodes exchange (January 2011) and reminimisation of laser sampling error at $20 \mathrm{kHz}$ after beam splitter exchange (March 2011). Instrument realignments are shown with dotted lines.

to 0.0007 and 0.0012 in the lamp and solar LSE estimates for the 20 and $40 \mathrm{kHz}$ data respectively. The reason for this is not understood, but is not believed to be due to detector nonlinearity. Lamp LSE estimates suggest a slight increase in the magnitude of the LSE between July and October 2010 at 10 and $20 \mathrm{kHz}$ sample rates. Unfortunately, the very limited number of lamp measurements precludes any real validation of the LSE tendency observed in the $20 \mathrm{kHz}$ solar LSE time series between July 2010 and January 2011.

We turn now to the LSE estimates for the ECL05 laser board and v02 diodes, (post January 2011). The contrast between the magnitude and sample rate dependence of the ECL03 and ECL05 boards is very clear in the lamp LSE time series (Fig. 5). Since installation of the ECL05 board in January 2011 the magnitude of the LSE correction derived from lamp measurements has remained less than 0.0002 at all sample rates during periods when the sampling error was minimised. The LSE correction at the sample rate for which the error was minimised $(20 \mathrm{kHz})$ is less than $5 \times 10^{-5}$. Even when the sampling error minimisation was not optimal (e.g. three days in April 2012 following the laser exchange) the sampling error was still 0.0005 or less for all sample rates. This is an order of magnitude improvement on the LSE for the ECL03 board.

The ECL05 LSE at $20 \mathrm{kHz}$ inferred from solar measurements prior to the beam splitter exchange in March 2011 are slightly larger than the corresponding lamp estimates. Following the beam splitter exchange there is an asymmetry in the LSE inferred for forward (LSE 0) and reverse (LSE 
Table 1. Comparison of laser sampling error estimates derived from lamp and solar measurements acquired at different sample rates (Rate, $\mathrm{kHz}$ ) using the Lauder 125HR with the ECL03 laser board. All tabulated lamp data were acquired using the NDACC filter. Lamp GPR estimates have been scaled by $\gamma$ to convert them to sampling errors (see Sect. 3.4 for details). Bracketed (f) and (r) refer to forward and reverse scans. In general there is excellent agreement between the magnitude and sample rate dependence of the sampling errors inferred from lamp spectra using the resampling algorithm (LSE) and the GPR estimates ( $\gamma \times$ GPR). There is also excellent agreement between the $10 \mathrm{kHz}$ sampling error estimates inferred using solar and lamp measurements acquired within a few days of one another. However, significant differences can be seen between solar and lamp LSE estimates for the 20 and $40 \mathrm{kHz}$ sample rates.

\begin{tabular}{|c|c|c|c|c|c|}
\hline \multirow{2}{*}{$\begin{array}{l}\text { Rate } \\
(\mathrm{kHz})\end{array}$} & \multicolumn{3}{|c|}{ Lamp } & \multicolumn{2}{|c|}{ Solar } \\
\hline & Date & $\gamma \times \mathrm{GPR}$ & LSE & Date & LSE \\
\hline 10 & $19 / 07 / 2010$ & 0.002 & $\begin{array}{r}-0.0020(\mathrm{f}) \\
0.0020(\mathrm{r})\end{array}$ & $04 / 08 / 2010$ & $\begin{array}{r}0.0025(\mathrm{f}) \\
-0.0026(\mathrm{r})\end{array}$ \\
\hline 20 & & 0.0001 & $\begin{array}{l}0.0001(\mathrm{f}) \\
0.0001(\mathrm{r})\end{array}$ & & $\begin{array}{r}0.0006(\mathrm{f}) \\
-0.0008(\mathrm{r})\end{array}$ \\
\hline 40 & & 0.006 & $\begin{array}{r}0.0060(\mathrm{f}) \\
-0.0056(\mathrm{r})\end{array}$ & & \\
\hline 10 & 08/10/2010 & 0.0022 & $\begin{array}{r}-0.0022(\mathrm{f}) \\
0.0024(\mathrm{r})\end{array}$ & $02 / 10 / 2010$ & $\begin{array}{r}0.0025(\mathrm{f}) \\
-0.0027(\mathrm{r})\end{array}$ \\
\hline 20 & & 0.0004 & $\begin{array}{r}-0.0002(\mathrm{f}) \\
0.0004(\mathrm{r})\end{array}$ & & $\begin{array}{r}0.0009(\mathrm{f}) \\
-0.0011(\mathrm{r})\end{array}$ \\
\hline 40 & & 0.0053 & $\begin{array}{r}0.0053(\mathrm{f}) \\
-0.0050(\mathrm{r})\end{array}$ & & $\begin{array}{r}-0.0041(\mathrm{f}) \\
0.0038(\mathrm{r})\end{array}$ \\
\hline 10 & $18 / 01 / 2011$ & 0.0025 & $\begin{array}{r}-0.0023(\mathrm{f}) \\
0.0023(\mathrm{r})\end{array}$ & & \\
\hline 20 & & 0.0005 & $\begin{array}{r}-0.0004(\mathrm{f}) \\
0.0005(\mathrm{r})\end{array}$ & 09/01/2011 & $\begin{array}{r}0.0009(\mathrm{f}) \\
-0.0009(\mathrm{r})\end{array}$ \\
\hline 40 & & 0.0050 & $\begin{array}{r}0.0049(\mathrm{f}) \\
-0.0046(\mathrm{r})\end{array}$ & & \\
\hline 10 & $19 / 01 / 2011$ & 0.0023 & $\begin{array}{r}-0.0022(\mathrm{f}) \\
0.0024(\mathrm{r})\end{array}$ & & \\
\hline 20 & & 0.0005 & $\begin{array}{r}-0.0004(\mathrm{f}) \\
0.0005(\mathrm{r})\end{array}$ & $13 / 01 / 2011$ & $\begin{array}{r}0.0004(\mathrm{f}) \\
-0.0009(\mathrm{r})\end{array}$ \\
\hline 40 & & 0.0050 & $\begin{array}{r}0.0050(\mathrm{f}) \\
-0.0047(\mathrm{r})\end{array}$ & & \\
\hline
\end{tabular}

$\sim-0.0005)$ scans. Further study is required to determine the origin of these systematic differences between lamp and solar LSE estimates.

\section{LSE assumed in resampling}

The LSE (forward, reverse) of the $10 \mathrm{kHz}$ measurements is approximated by $(-0.0022,0.0023)$ prior to 18 December 2009 intervention and $(0.0024,-0.0026)$ post 15 January 2010 alignment. These values are determined from the mean of the solar LSE estimates for the two periods.

The discrepancy between lamp and solar LSE estimates at 20 and $40 \mathrm{kHz}$ poses something of a dilemma when defining the LSE to be used in resampling. To date, both datasets have been resampled using the LSE inferred from solar measurements. This might give an overly optimistic view of the resampling algorithm performance if the lamp LSE estimates are in fact better estimates of the true sampling errors. This is discussed further in Sect. 4.2 below. The $40 \mathrm{kHz}$ cycling solar data have been resampled assuming LSE of (-0.004, 0.004). The $20 \mathrm{kHz}$ data in the examples shown have been resampled assuming an LSE of $(0.0006,-0.0010)$ for the period February-March 2010, and an LSE of $(0.0009,-0.0010)$ in October 2010.

\subsubsection{Izaña 125HR time series}

The LSE time series for the Izaña 125HR from September 2008 to October 2011 is illustrated in Fig. 6, where the LSE of the $40 \mathrm{kHz}$ measurements were determined by using the regions $7290-7360 \mathrm{~cm}^{-1}$ (in most of the cases) and $10900-11300 \mathrm{~cm}^{-1}$. The beginning of new sub-periods with different LSE due to instrumental interventions or breakdowns are indicated with vertical dashed lines. The values of the LSE (forward, reverse) are determined from the mean estimated LSE of each period. The first resampling results of the solar data acquired with the original ECL02 laser board from September 2008 to March 2009 show large LSE 


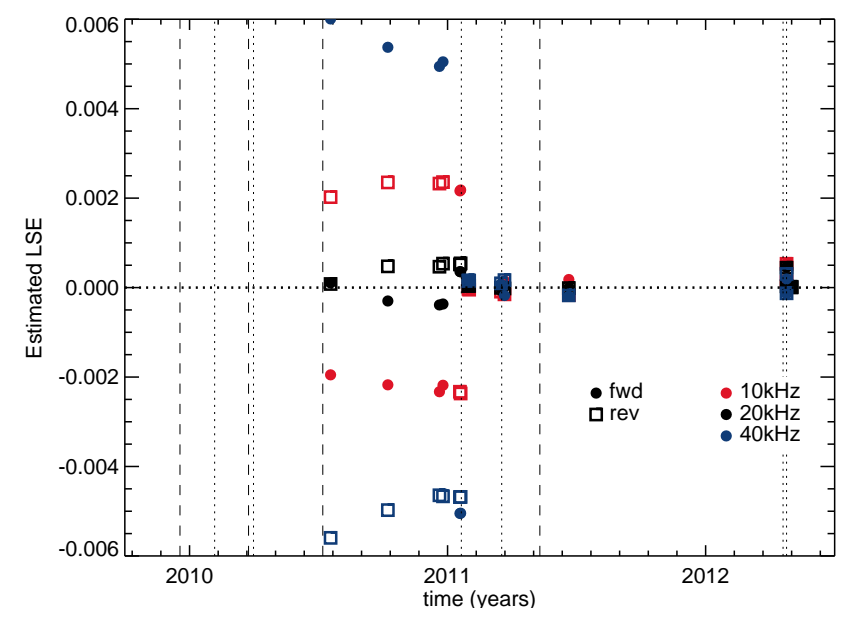

Fig. 5. Lauder 125HR LSE time series determined from analysis of tungsten lamp spectra acquired with narrow bandpass optical filters (NDACC, TCCON) since July 2010. The lamp sequences acquired in January 2011 have been artificially reshuffled for clarity: estimates acquired with the NDACC HF filter appear plotted just prior to 1 January 2011 , followed by two realisations with the TCCON filter. The TCCON filter has been used exclusively to monitor the LSE monitoring following the laser board exchange (20 January 2011). Instrument changes are shown with vertical lines (refer to the caption of Fig. 4 for details).

estimates of about $(0.0094,-0.0068)$. The electrical lowpass-filter change in March 2009 did not influence the LSE, whereas the laser board exchange (ECL03 laser board) and red-filter installation in November/December 2009 reduced the LSE to $(0.0046,-0.0036)$. The next stepwise changes in the LSE are evident for instrument interventions due to loss of power and very cold temperatures $\left(<0^{\circ} \mathrm{C}\right)$ in the container (January-March 2010) and the electrical low-passfilter change in May 2010. We see a clear contrast between the LSE estimates for the ECL02/ECL03 and ECL04 board, which was installed with v02 diodes in November 2010. Since that installation the magnitude of the LSE remains at $(0.0020,-0.0001)$, where the residual LSE might be due to perturbations of the zero baseline that we clearly see for forward scans. There are no lamp measurements available at Izaña to provide an independent check on the LSE derived from the solar measurements.

\subsection{Retrieval case studies}

\subsubsection{Lauder cycling sample rate experiments}

Figure 7 illustrates the typical results obtained by resampling 10,20 and $40 \mathrm{kHz}$ time series for the 5 days of cycling sample rate experiments undertaken at Lauder in October 2010. Retrievals at 10 and $40 \mathrm{kHz}$ differ from the $20 \mathrm{kHz}$ retrievals by -0.2 and $0.5 \%$ respectively. After resampling, the discrepancy between sample rates is reduced to less than $0.1 \%$. Approximately $75 \%$ of the difference between original and

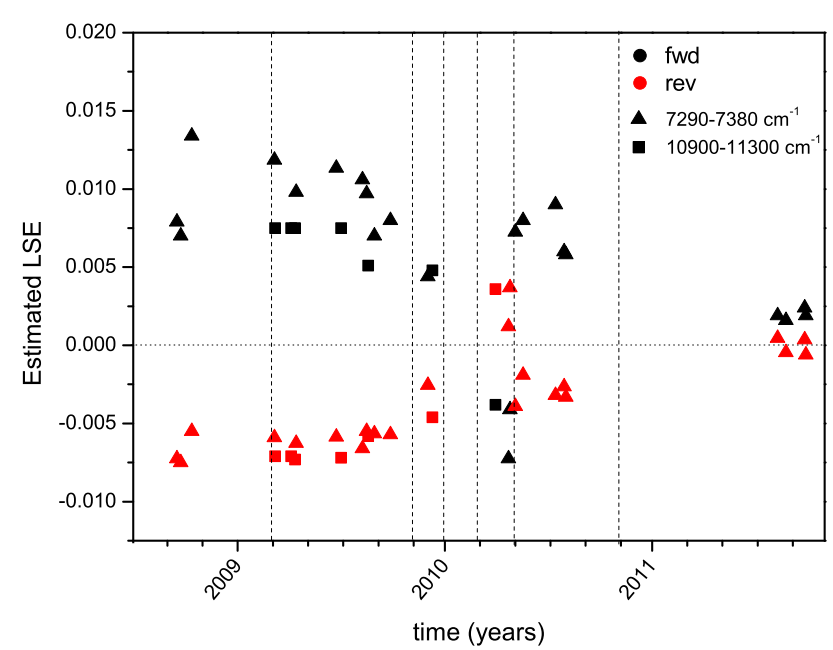

Fig. 6. Izaña LSE time series from 2008 to 2011 analysed using the regions $7290-7360 \mathrm{~cm}^{-1}$ (triangles) and 10900 $11300 \mathrm{~cm}^{-1}$ (squares). The LSE which minimises the mean intensity is determined by a polynomial fit around the minimum of a curve like those shown in Fig. 3. Forward (black) and reverse scans (red) vary in magnitude and sign. The beginning of new sub-periods with different LSE due to instrumental interventions or breakdowns are indicated with vertical dashed lines: electrical low-pass-filter change (March 2009), laser board exchange and red-filter installation (November/December 2009), loss of power and very cold temperatures (January-March 2010), electrical low-pass-filter change (May 2010), installation of ECL04 laser board and v02 diodes (November 2010).

resampled $X_{\mathrm{CO}_{2}}$ retrievals is due to changes in the $\mathrm{O}_{2}$ retrieval.

The Lauder $125 \mathrm{HR} X_{\text {air }}$ time series during early 2010 is illustrated in Fig. 8. During this period we changed from 10 to $20 \mathrm{kHz}$ sample rate for routine solar measurements, with experiments cycling between 10 and $20 \mathrm{kHz}$ sample rate on several days. To characterise the change in $X_{\text {air }}$ retrievals due to the LSE (only), we consider a restricted range of zenith angles. In this way, air-mass-dependent retrieval biases (due to errors in spectroscopy and/or instrument alignment) do not mask the LSE signature. Again, the raw retrievals show a $0.2 \%$ difference in $X_{\text {air }}$ retrieved from spectra acquired at 10 and $20 \mathrm{kHz}$ during common time intervals. After resampling the $10 \mathrm{kHz}$ data, the difference with respect to the original $20 \mathrm{kHz}$ data is reduced to $0.1 \%$. The agreement between the $X_{\text {air }}$ distributions for the resampled data at 10 and $20 \mathrm{kHz}$ is excellent. $X_{\text {air }}$ values for the median, upper and lower fourths of the two distributions differ by $0.03 \%$ or less.

\subsubsection{Izaña $X_{\text {air }}$ and $X_{\mathrm{CO}_{2}}$ time series}

The Izaña $125 \mathrm{HR}$ time series of daily mean $X_{\text {air }}$ and $X_{\mathrm{CO}_{2}}$ from 2008 to 2011 acquired at solar zenith angles between 40 and $60^{\circ}$ are illustrated in Figs. 9 and 10. The resampled measurements are displayed as red dots, whereas the black dots show the original measurements. 

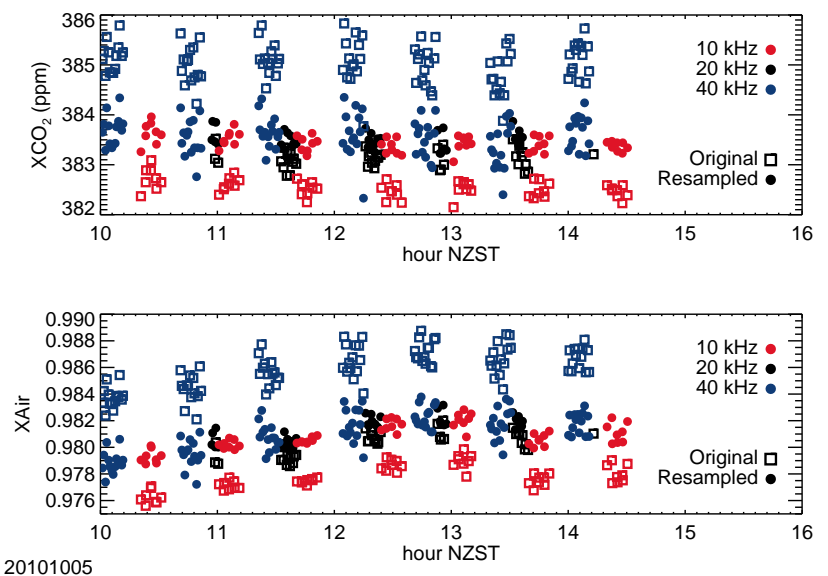

Fig. 7. Cycling sample rate experiments undertaken on the Lauder 125HR with the ECL03 version of the laser board on 5 October 2010. Retrievals from original interferograms are illustrated with squares for three sample rates $10 \mathrm{kHz}$ (red), $20 \mathrm{kHz}$ (black) and $40 \mathrm{kHz}$ (blue) showing a marked sample rate dependence. Retrievals from resampled interferograms are shown with filled circles. Resampling reduces discrepancies between the sample rates to less than $0.1 \%$.

The uncorrected Izaña $X_{\text {air }}$ time series in Fig. 9 shows seven distinct periods with different mean $X_{\text {air }}$, attributed to intentional modifications on the instrument, e.g. realignment of the interferometer, exchange of reference laser, change of relevant measurement settings and laser board exchange or adjustments. Rarely, in the course of strong storms, prolonged power breakdowns of the whole container occur at Izaña which result in very low temperature of the spectrometer. In addition, we find indications for an unexpected high bias of $X_{\text {air }}$ during the period before September 2008 (not shown), which need to be investigated further. In the end of 2010, the new ECL04 Bruker laser sampling unit was installed, which introduced a significant $0.35 \%$ reduction of $X_{\text {air. }}$. Henceforward, it is assumed that these measurements are free of ghosts and provide a reference $X_{\text {air }}$ value. By the use of resampled data, this difference is reduced to $0.2 \%$. We attribute the remaining discrepancy to changes in interferometer alignment. In addition we see a discontinuity for the resampled measurements between March 2009 and May 2010. This might be due to changes of the electrical low-pass-filter settings in this period, but has to be investigated further. Note in the KIT interferogram preprocessing the forward and reverse scans are corrected for their respective sampling errors, then coadded and transformed to generate one spectrum per forward/reverse pair, so the reduction in the discrepancy between forward and reverse scans on resampling has not been characterised explicitly.

The $X_{\mathrm{CO}_{2}}$ time series shows a reduction of $X_{\mathrm{CO}_{2}}$ for resampled measurements. As mentioned before, most of this difference is due to changes in the $\mathrm{O}_{2}$ retrieval. For measurements at the beginning of the time series in 2008 , the
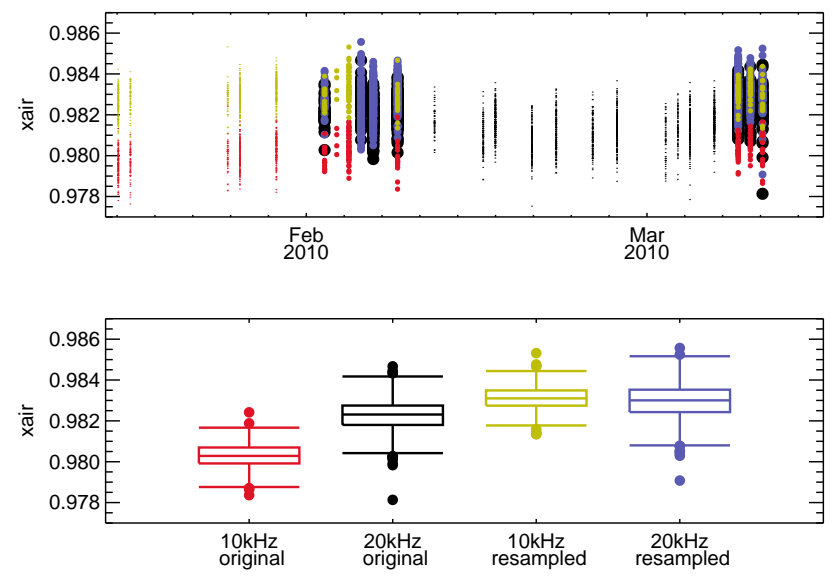

Fig. 8. The upper panel shows Lauder $125 \mathrm{HR} X_{\text {air }}$ retrievals from original 10 and $20 \mathrm{kHz}$ data (red and black points respectively), acquired between 18 January and 18 March 2010 at solar zenith angles of $40-60^{\circ}$. The corresponding resampled $10 \mathrm{kHz}$ retrievals are shown in yellow and resampled $20 \mathrm{kHz}$ retrievals are shown in purple. To aid comparison, the lower panel shows box and whisker summaries of the distribution of $X_{\text {air }}$ retrieved at 10 and $20 \mathrm{kHz}$ before and after resampling (these summaries are for the measurement subintervals when both sample rates were used, and are shown with the larger sized symbols in the upper panel). There is clear evidence of a low bias of $0.2 \%$ in the original $10 \mathrm{kHz} X_{\text {air }}$ retrievals, relative to the $20 \mathrm{kHz}$ retrievals. After resampling, the distribution of $X_{\text {air }}$ retrieved at $10 \mathrm{kHz}$ lies entirely within the range of the $20 \mathrm{kHz} X_{\text {air }}$ distribution, and central $50 \%$ of two distributions (the boxes) are essentially co-incident (the medians differ by $0.01 \%$ and the lower fourths differ by $0.03 \%$ ).

difference between retrievals from uncorrected and resampled spectra is about $0.2 \%$. Smaller differences $(0.03 \%)$ are found in the period before the laser board exchange in 2010 .

\subsubsection{Retrieval sensitivities inferred from the resampled time series}

At Lauder retrieval differences for $X_{\text {air }}$ and $X_{\mathrm{CO}_{2}}$ are only weakly dependent on air mass for zenith angles less than $80^{\circ}$. For example, for the $10 \mathrm{kHz}$ dataset, $X_{\text {air }}$ retrieval biases vary by 0.0005 (increasing with solar zenith angle) for zenith angles between 20 and $80^{\circ} . X_{\mathrm{CO}_{2}}$ varies by $0.05 \mathrm{ppm}$ over the same range of zenith angles. There is evidence of more marked differences (0.001-0.002) in $X_{\text {air }}$ at high zenith angles, but the impact in $X_{\mathrm{CO}_{2}}$ remains small. No marked temporal signatures (other than that associated with the changes in the LSE estimates applied in resampling) were evident in the time series of retrieval differences, although admittedly the $10 \mathrm{kHz}$ sequence only spans a year and is generally very sparsely sampled.

To first order the retrieval sensitivity for the Lauder 125HR, $X_{\text {air }}$ and $X_{\mathrm{CO}_{2}}$ measurements can therefore each be characterised by a single number. We estimate an $X_{\text {air }}$ bias of 0.0012 and $X_{\mathrm{CO}_{2}}$ bias of $0.36 \mathrm{ppm}$ per $0.001 \mathrm{LSE}$ 


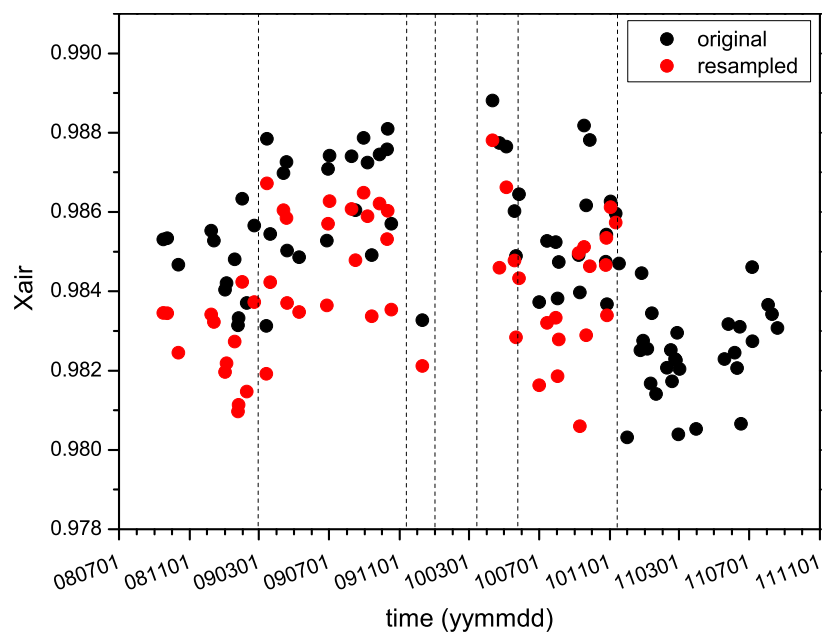

Fig. 9. Izaña $125 \mathrm{HR}$ time series of daily mean $X_{\text {air }}$ from 2008 to 2011 for original and resampled measurements. The beginning of new sub-periods with different $X_{\text {air }}$ due to instrumental interventions or breakdowns are indicated with vertical dashed lines: electrical low-pass-filter change (March 2009), laser board exchange and red-filter installation (November/December 2009), loss of power and very cold temperatures (January-March 2010), electrical lowpass-filter change (May 2010), installation of ECL04 laser board and v02 diodes (November 2010). Resampling reduces the discrepancy to $\sim 0.2 \%$.

for LSE in the range $0-0.004$. We infer that the error due to the discrepancy in lamp and solar LSE estimates is small for the Lauder $125 \mathrm{HR}$ data $(0.1 \%)$, but this is still significant compared to the TCCON inter-station comparability target $(0.1 \%)$.

For Izaña the bias of $X_{\text {air }}$ and $X_{\mathrm{CO}_{2}}$ per LSE with the $40 \mathrm{kHz}$ sample rate is estimated assuming an effective LSE equal to the mean LSE of the forward and reverse scans. The bias of $X_{\text {air }}$ per 0.001 LSE is about 0.0003 , whereas a $X_{\mathrm{CO}_{2}}$ bias of $0.11 \mathrm{ppm}$ per $0.001 \mathrm{LSE}$ is determined for $|\mathrm{LSE}|$ in the range of $0.001-0.01$.

These retrieval sensitivities inferred from the resampled time series at Lauder and Izaña can be compared with sensitivities of 0.00085 and 0.28 ppm per 0.001 LSE for $X_{\text {air }}$ and $X_{\mathrm{CO}_{2}}$ derived from a reanalysis of the Messerschmidt et al. (2010) datasets (Sherlock et al., 2011). The retrieval sensitivity to LSE inferred from the three datasets differ significantly: by a factor $\sim 1.3-4$. Taken at face value, this result implies a site-independent relationship between $X_{Y}$ and the LSE (a key assumption of the empirical correction proposed by Messerschmidt et al., 2010) cannot generally be assumed. Such a discrepancy could be due to differences in the instrument spectral response and/or differences in the line-of-sight abundances of the target gases and interfering absorbers (particularly $\mathrm{H}_{2} \mathrm{O}$ ), which depend on site altitude and environmental conditions. Given the magnitude of the discrepancy, this merits some further study.

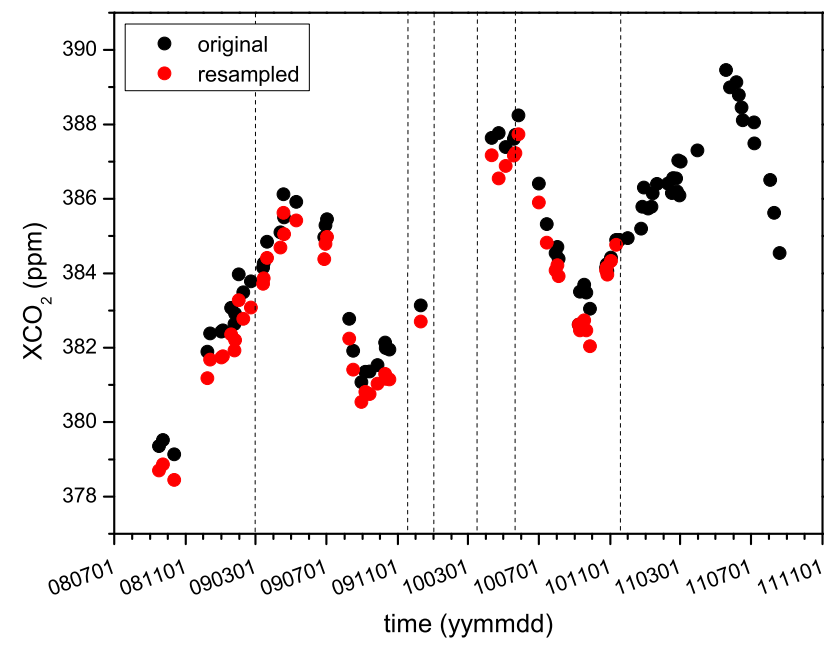

Fig. 10. Izaña $125 \mathrm{HR}$ time series of daily mean $X_{\mathrm{CO}_{2}}$ from 2008 to 2011 for original and resampled measurements. The beginning of new sub-periods with different $X_{\text {air }}$ due to instrumental interventions or breakdowns are indicated with vertical dashed lines: electrical low-pass-filter change (March 2009), laser board exchange and red-filter installation (November/December 2009), loss of power and very cold temperatures (January-March 2010), electrical lowpass-filter change (May 2010), installation of ECL04 laser board and v02 diodes (November 2010). The plot indicates a reduction of $X_{\mathrm{CO}_{2}}$ for resampled measurements, which is mostly due to changes in the $\mathrm{O}_{2}$ retrieval.

\section{Conclusions}

We have described and demonstrated a two-step procedure which enables the laser sampling error (LSE) history to be characterised through time and which enables sampling errors to be corrected at the source by resampling the interferograms.

LSE estimates derived using the resampling method are in good agreement with sampling errors inferred from lamp GPR measurements at Lauder. The maximum difference observed for the $20 \mathrm{kHz}$ sample rate is 0.0007 , implying a limit to correction accuracy of $0.08 \%$. The corresponding limit inferred for the $40 \mathrm{kHz}$ sample rate is slightly higher, at $0.14 \%$.

Sampling histories at Lauder and Izaña show clear changes in LSE due to instrument interventions and changes in the measurement sample rate. Similar histories are to be expected at other TCCON sites. Resulting retrieval biases are in the range of $0.2-0.5 \%$, and are time and site dependent. After resampling, discrepancies in the retrieved $X_{\text {air }}$ and $X_{\mathrm{CO}_{2}}$ due to instrument interventions and changes in the measurement sample rate are reduced to $0.1 \%$ or less at Lauder and $0.2 \%$ at Izaña. In the latter case, coincident changes in interferometer alignment may also contribute to the residual difference.

These errors could not have been adequately corrected for using the empirical method proposed by Messerschmidt et al. 
(2010), first and foremost because no suitable time series of lamp GPR characterisations exist for these or other TCCON sites. Inferred retrieval sensitivities to LSE also differ by a factor of 1.3-4, indicating that a site-independent relationship between $X_{Y}$ and the LSE cannot generally be assumed.

Although not shown explicitly here, laser sampling errors affect the estimated DMF of other TCCON target gases $\left(\mathrm{CH}_{4}, \mathrm{~N}_{2} \mathrm{O}, \mathrm{CO}\right)$ in a similar manner. This is because the bias in $X_{Y}$ for these gases is primarily driven by the bias in the $\mathrm{O}_{2}$, as it is for $X_{\mathrm{CO}_{2}}$.

In the next step, corrections must be determined and applied to historical data at all TCCON sites. This should improve the inter-station comparability of the TCCON, and this will be characterised using the $\mathrm{O}_{2}$ internal network standard.

Acknowledgements. We thank Axel Keens of Bruker Optik GmbH for many useful discussions through the course of this work. From 2004 to 2011 the Lauder TCCON programme was funded by the New Zealand Foundation of Research Science and Technology contracts CO1X0204, CO1X0703 and CO1X0406. Since 2011 the programme has been funded by NIWA's Atmosphere Research Programme 3 (2011/13 Statement of Corporate Intent). E. Sepúlveda is funded by a pre-doctoral fellowship from the Spanish Ministry of Education. We thank the Goddard Space Flight Center for providing the temperature and pressure profiles of the National Centers for Environmental Prediction via the automailer system. We acknowledge support by the Deutsche Forschungsgemeinschaft and Open Access Publishing Fund of the Karlsruhe Institute of Technology.

The service charges for this open access publication have been covered by a Research Centre of the Helmholtz Association.

Edited by: H. Worden

\section{References}

Chevallier, F., Deutscher, N. M., Conway, T. J., Ciais, P., Ciattaglia, L., Dohe, S., Fröhlich, M., Gomez-Pelaez, A. J., Griffith, D., Hase, F., Haszpra, L., Krummel, P., Kyrö, E., Labuschagne, C., Langenfelds, R., Machida, T., Maignan, F., Matsueda, H., Morino, I., Notholt, J., Ramonet, M., Sawa, Y., Schmidt, M., Sherlock, V., Steele, P., Strong, K., Sussmann, R., Wennberg, P., Wofsy, S., Worthy, D., Wunch, D., and Zimnoch, M.: Global $\mathrm{CO}_{2}$ fluxes inferred from surface air-sample measurements and from TCCON retrievals of the CO2 total column, Geophys. Res. Lett., 38, L24810, doi:10.1029/2011GL049899, 2011.

Gisi, M., Hase, F., Dohe, S., and Blumenstock, T.: Camtracker: a new camera controlled high precision solar tracker system for FTIR-spectrometers, Atmos. Meas. Tech., 4, 47-54, doi:10.5194/amt-4-47-2011, 2011.

Griffith, D., Jones, N., McNamara, B., Paton-Walsh, C., Bell, W., and Bernardo, C.: Intercomparison of ground-based solar FTIR measurements of atmospheric gases at Lauder, New Zealand, J. Atmos. Ocean. Tech., 20, 1138-1153, 2003.
Guelachvili, G.: Spectrometric Techniques, chap. 1. Distortions in Fourier spectra and diagnosis, 1-61, Academic Press, ISBN 9780127104027, 1981.

Hase, F., Blumenstock, T., and Paton-Walsh, C.: Analysis of the instrumental line shape of high-resolution Fourier Transform IR spectrometers with gas cell measurements and new retrieval software, Appl. Optics, 38, 3417-3422, 1999.

Hase, F., Hannigan, J., Coffey, M., Goldman, A., Hopfner, M., Jones, N., Rinsland, C., and Wood, S.: Intercomparison of retrieval codes used for the analysis of high-resolution, groundbased FTIR measurements, J. Quant. Spectrosc. Ra., 87, 25-52, doi:10.1016/j.jqsrt.2003.12.008, 2004.

Keppel-Aleks, G., Wennberg, P. O., Washenfelder, R. A., Wunch, D., Schneider, T., Toon, G. C., Andres, R. J., Blavier, J.-F., Connor, B., Davis, K. J., Desai, A. R., Messerschmidt, J., Notholt, J., Roehl, C. M., Sherlock, V., Stephens, B. B., Vay, S. A., and Wofsy, S. C.: The imprint of surface fluxes and transport on variations in total column carbon dioxide, Biogeosciences, 9, 875891, doi:10.5194/bg-9-875-2012, 2012.

Learner, R., Thorne, A., and Brault, J.: Ghosts and artifacts in Fourier-Transform spectrometry, Appl. Optics, 35, 2947-2954, 1996.

Messerschmidt, J., Macatangay, R., Notholt, J., Petri, C., Warneke, T., and Weinzierl, C.: Side by side measurements of $\mathrm{CO}_{2}$ by ground-based Fourier transform spectrometry (FTS), Tellus B, 62, 749-758, doi:10.1111/j.1600-0889.2010.00491.x, 2010.

Messerschmidt, J., Geibel, M. C., Blumenstock, T., Chen, H., Deutscher, N. M., Engel, A., Feist, D. G., Gerbig, C., Gisi, M., Hase, F., Katrynski, K., Kolle, O., Lavrič, J. V., Notholt, J., Palm, M., Ramonet, M., Rettinger, M., Schmidt, M., Sussmann, R., Toon, G. C., Truong, F., Warneke, T., Wennberg, P. O., Wunch, D., and Xueref-Remy, I.: Calibration of TCCON column-averaged $\mathrm{CO}_{2}$ : the first aircraft campaign over European TCCON sites, Atmos. Chem. Phys., 11, 10765-10777, doi:10.5194/acp-11-10765-2011, 2011.

Miller, C. E., Crisp, D., DeCola, P. L., Olsen, S. C., Ran derson, J. T., Michalak, A. M., Alkhaled, A., Rayner, P., Jacob, D. J., Suntharalingam, P., Jones, D. B. A., Denning, A. S., Nicholls, M. E., Doney, S. C., Pawson, S., Boesch, H., Connor, B. J., Fung, I. Y., O'Brien, D., Salawitch, R. J., Sander, S. P., Sen, B., Tans, P., Toon, G. C., Wennberg, P. O., Wofsy, S. C., Yung, Y. L., and Law, R. M.: Precision requirements for space-based data, J. Geophys. Res.-Atmos., 112, D10314, doi:10.1029/2006JD007659, 2007.

Sherlock, V., Messerschmidt, J., and Hase, F.: Ghost working group update 17/01/2011, Internal TCCON technical report, available on request, 2011.

WMO: 15th WMO/IAEA Meeting of Experts on Carbon Dioxide, Other Greenhouse Gases and Related Tracers Measurements Techniques, GAW Report No. 194, WMO TD No. 1553, 330 pp., 2011.

Wunch, D., Toon, G., Blavier, J.-F., Notholt, R. W. J., Connor, B., Griffith, D., Sherlock, V., and Wennberg, P.: The Total Carbon Column Observing Network, Philos. T. Roy. Soc. A, 369, 20872112, doi:10.1098/rsta.2010.0240, 2011. 